\title{
Objectively Measured Physical Activity Is Negatively Associated with Plasma Adiponectin Levels in Minority Female Youth
}

\author{
B. Adar Emken, ${ }^{1}$ Joyce Richey, ${ }^{2}$ Britni Belcher, ${ }^{1}$ Ya-Wen Hsu, ${ }^{1}$ and Donna Spruijt-Metz ${ }^{1}$ \\ ${ }^{1}$ Department of Preventive Medicine, Keck School of Medicine, University of Southern California, Los Angeles, CA 90089, USA \\ ${ }^{2}$ Department of Physiology and Biophysics, Keck School of Medicine, University of Southern California, Los Angeles, CA 90089, USA
}

Correspondence should be addressed to Donna Spruijt-Metz, dmetz@usc.edu

Received 26 March 2010; Revised 9 June 2010; Accepted 10 June 2010

Academic Editor: Paul L. Hofman

Copyright $\odot 2010$ B. Adar Emken et al. This is an open access article distributed under the Creative Commons Attribution License, which permits unrestricted use, distribution, and reproduction in any medium, provided the original work is properly cited.

\begin{abstract}
Objective. To evaluate the relationship between adiponectin and physical activity (PA) in minority female youth. Methods. Plasma adiponectin was measured in 39 females (mean age $9.2 \pm 0.9$ years; 30 Latina, 9 African-American; 56\% overweight). PA was assessed by accelerometry. Mean minutes per day spent in daily PA (DPA) ( $\geq 3$ metabolic equivalents (METs)), moderate PA (MPA)(4-7 METs), vigorous PA (VPA)( $\geq 7$ METs), and moderate-to-vigorous PA (MVPA)( $\geq 4$ METs) were calculated. The association between adiponectin and PA, controlling for age, fat weight, lean weight, and insulin sensitivity (SI) was analyzed using linear regression. Results. Adiponectin correlated with fat weight $(r=-0.43, P<.01)$ and SI $(r=0.52, P<.01)$. Minutes spent in DPA $(\beta=-0.40, P=.02)$, MPA $(\beta=-0.36, P=.04)$, or MVPA $(\beta=-0.37, P=.03)$ were predictors of adiponectin in the adjusted model. Conclusions. Higher PA levels were related to lower adiponectin levels. Potential mechanisms include upregulation of adiponectin receptors or an increase in high-molecular weight adiponectin with increasing PA.
\end{abstract}

\section{Introduction}

Adiponectin is a protein that is secreted by adipose tissue. Unlike other adipose-derived hormones such as leptin, adiponectin levels decrease with increasing body mass index (BMI) and are lower in obese individuals [1]. High levels of adiponectin could be beneficial because adiponectin promotes insulin sensitivity and has anti-inflammatory effects [2]. Physical activity (PA) may increase levels of adiponectin; however, results are mixed (for a review, see [3]).

An increasing number of children in the United States are overweight or obese, particularly in minority populations [4]. Adiponectin levels are lower in overweight or obese children compared to normal weight counterparts, and males generally have lower adiponectin levels than females [5]. Low levels of adiponectin can be a predictor of health outcomes such as cardiovascular disease, cancer, and type 2 diabetes in adults [6-8], and consequently low adiponectin levels during childhood or adolescence could have negative long-term health effects.

In children and adolescents, studies have reported increases $[9,10]$, no change $[11,12]$, or decreases $[13,14]$ in adiponectin with increasing PA. In a small study evaluating a 12 -week combined diet and physical activity intervention in 15 obese adolescents, the intervention resulted in increased adiponectin levels and decreased percent body fat [9]. However, the study involved a combined intervention and the effect of the decrease in body fat on adiponectin levels was not taken into account. Likewise, a study of 26 obese Korean adolescents showed an increase in adiponectin following a 6-week jump rope intervention, without adjusting for decreases in body fat [10]. Other studies report no relationship between PA and adiponectin in children and adolescents, including a large cross-sectional study of FrenchCanadian youth using a self-reported measure of PA [11] and a 12-week PA intervention involving supervised physical activity three times a week in overweight Greek adolescents [12]. Interestingly, a study of mainly Caucasian children in England aged 5-8 years found that adiponectin levels decreased with increasing PA measured by accelerometry, independent of percent body fat and that this effect was stronger in females than males [13]. An additional study in adolescents using an activity questionnaire found a trend towards decreased adiponectin with increasing PA [14]. 
The direction of the relationship between adiponectin and PA thus remains unclear, and the strength of the relationship may differ by gender [13]. In addition, no studies have examined the relationship between physical activity and adiponectin levels in minority children, although one study did find a relationship between increased physical fitness (measured by $\mathrm{VO}_{2 \max }$ ) and decreased adiponectin in Hispanic and Asian-American children [15]. The purpose of this study was to evaluate if adiponectin levels are associated with PA measured by accelerometry independent of adiposity in Latina and African-American female youth.

\section{Methods}

2.1. Participants. Participants were members of the TRANSITIONS Study, a longitudinal study of psychosocial and physiological determinants of PA in Latina and AfricanAmerican females during the pubertal transition. Participants included in this study were those who had completed their first annual visit in the TRANSITIONS Study by June 2009 and had accelerometry and adiponectin data from that visit. TRANSITIONS participants were recruited from Los Angeles County through participating physicians and clinics, churches, schools, and community centers. Inclusion criteria were: female gender, Tanner stage 1 or 2, nonmenstruating, between the ages of 8 and 11, and Hispanic or AfricanAmerican ethnicity (both parents and all four grandparents were Hispanic (Mexican or Central American) or AfricanAmerican). Participants were also required to meet one of the following criteria: (1) age- and gender-specific BMI $\geq 85$ th percentile of the Centers for Disease Control and Prevention (CDC) reference data [16], (2) one overweight parent (BMI $\geq 25$ ), or (3) one parent with diagnosed diabetes. Exclusion criteria were intake of medications known to influence body composition, insulin action, or insulin secretion, meeting diagnostic criteria for diabetes (assessed by fasting plasma glucose $\geq 126 \mathrm{mg} / \mathrm{dL}$ during a screening visit), or a previous diagnosis of a major illness or disease. Parents and children were provided with detailed written and verbal information about the study in their primary language and informed written consent from the parent and assent from the child was obtained. The study was approved by the Institutional Review Board of the University of Southern California (USC).

2.2. Study Procedures. All procedures took place during an overnight visit to the USC General Clinical Research Center (GCRC). Upon arrival to the GCRC at approximately 13:00, participants and their parents were asked to complete demographic and psychosocial questionnaires. Afterwards a licensed pediatric health care provider performed a complete medical examination and health history on the participant, including verification of Tanner stage. Participants were given a standardized dinner and a snack before 20:00 and were instructed to refrain from food or drink other than water after 20:00. Participants spent the night in the GCRC and the overnight fast was verbally verified with the participant and parent in the morning. At 08:00, a fasting blood draw was completed and followed by initiation of the frequently sampled intravenous glucose tolerance test (FSIVGTT). At time 0 , glucose (25\% dextrose, $0.3 \mathrm{~g} / \mathrm{kg}$ body weight) was administered intravenously. Blood samples were collected at time points $-15,-5,2,4,8,19,22,30,40,50$, 70,100 , and 180 minutes. Insulin ( 0.02 units/kg body weight, Humulin R (regular insulin for human injection); Eli Lilly, Indianapolis, IN) was injected intravenously at 20 minutes. Following completion of the FSIVGTT, participants were given lunch and completed measures of body composition. At discharge, participants were provided with an accelerometer, instructed on how to wear it, and asked to wear the accelerometer for the ensuing 7 days. Participants were called several times during the week to remind them to wear their accelerometer. At the end of the week, study staff retrieved the accelerometer from participant's homes.

2.3. Anthropometric Measurements. Weight and height were measured three times to the nearest $0.1 \mathrm{~kg}$ and $0.1 \mathrm{~cm}$, respectively, using a beam medical scale and wall-mounted stadiometer. The mean of the three measurements was used to calculate BMI and BMI percentiles for age and gender using publicly available SAS syntax from CDC (available at: http://www.cdc.gov/nccdphp/dnpa/growthcharts/ resources/index.htm). Children were classified as overweight (including obese) if they had an age- and gender-specific $\mathrm{BMI} \geq 85$ th percentile of the CDC reference data [16]. Total body composition was measured by air displacement plethysmography (BodPod; Life Measurement Instruments, Concord, CA). This system uses computerized pressure sensors to determine body volume and body density through air displacement. BodPod provides an accurate measure of body fat and lean body mass in children without dunking, pinching, exposure to electric currents, or radiation, and has been validated in children against hydrostatic weighing, dual energy X-ray absorptiometry (DEXA), and multicompartment models $[17,18]$.

2.4. Clinical Measurements. Fasting blood samples were assayed for adiponectin, insulin, and glucose. All samples were collected in vacutainer tubes and stored on ice until centrifugation, after which plasma was separated and stored at $-80^{\circ} \mathrm{C}$ until analysis. Adiponectin concentration $(\mu \mathrm{g} / \mathrm{ml})$ was measured with an enzyme-linked immunosorbent assay (ELISA) kit (Millipore, St. Charles, MO). The intra- and interassay coefficients of variation $(\mathrm{CV})$ were $7.4 \%$ and $8.4 \%$, respectively. Insulin was measured with an ELISA (Millipore, St. Charles, MO). Glucose was measured on-line with an automated glucose analyzer (Yellow Springs Instrument, Yellow Springs, OH) using the glucose oxidase method. All samples were assayed in duplicate.

The results of the FSIVGTT were used to determine insulin sensitivity (SI) and acute insulin response to glucose (AIRg) using the minimal model [19] (MINMOD MILLENIUM 2002 computer program, Version 5.16; Richard Bergman, Los Angeles, CA [20]). Insulin sensitivity is a measure of insulin-stimulated glucose uptake and AIRg characterizes the first phase of insulin secretion after glucose administration. 
2.5. Accelerometry. A biaxial accelerometer (Actigraph GT1M, Pensacola, FL) was used to objectively measure physical activity. Participants were instructed to wear the device on their right hip during waking hours (with the exception of time spent in bathing or swimming activities) for the 7 days immediately following the visit to the GCRC. The accelerometers collected data in 15second epochs and the data were aggregated to 60-second epochs for use with the current activity cut points. Data was visually inspected for monitor malfunction (i.e., the monitor got wet) and improper wear (i.e., the participant wore the accelerometer overnight while sleeping) prior to data processing. Data processing was done using SAS 9.2. Minutes of wear and nonwear were calculated using a modification of a publicly available program (available at: http://www.riskfactor.cancer.gov/tools/nhanes_pam). Participants with $<4$ days of wear (10 hour/day) were asked to wear the accelerometer for an additional 7 day period. Data from the second wear was combined with that from the first wear to ensure sufficient days of wear.

Accelerometer data is presented as mean counts per minute (cpm) and as estimates of mean minutes spent in various activity levels. Mean cpm was calculated by dividing the total counts per day by the total minutes of wear per day. Mean minutes in the activity levels was calculated by summing each minute spent above the user-defined thresholds for moderate PA (MPA), vigorous PA (VPA), and moderate-to-vigorous PA (MVPA) and below the userdefined threshold for sedentary behavior (SB). The cutpoints for MPA and VPA were 4 and 7 metabolic equivalents (METs), respectively, cut points which have been proposed for children between the ages of 6 and 17 years [21]. In addition, daily PA (DPA) was assessed using a lower cut point (3 METs) [22] to capture a wider range of PA as well as to be comparable to studies using 3 METs as the cut point for MPA. The sedentary cut point was $\leq 100$ counts/minute [23] and has been validated in adolescents [24].

2.6. Statistical Analyses. Comparisons were made using chi-square for comparisons of differences in frequency (categorical variables), Student's $t$-test for comparison of means (continuous variables), and Pearson's correlation coefficient for linear correlations. Nonnormally distributed variables were log-transformed for use in linear correlation analysis. Several multiple linear regression analysis were performed to determine whether the association between adiponectin (dependent variable) and physical activity levels (independent variable) was significant after adjustment for the potential confounding factors of age, fat weight, lean weight, and SI. The potential confounders of age, fat weight, lean weight, and SI were included in all models and separate models were conducted for each physical activity variable (mean counts per minute, mean minutes spent in SB, MPA, VPA, MVPA, or DPA) to avoid multicolinearity caused by entering all the PA variables into one model. Calculations were done using SPSS 16.0 for Windows. Results were considered significant at $P<.05$.

\section{Results}

3.1. Subject Characteristics. Table 1 shows the characteristics of participants included in this study. Twenty-two participants (56\%) met criteria for overweight. When the overweight classification criteria proposed by the International Obesity Taskforce (IOTF) were applied, results were identical [25]. Of the 51 females who completed their first annual visit for the TRANSITIONS Study by June 2009, the 39 participants (30 Latina, 9 African American) who had an adiponectin measurement from that visit and at least four days of valid accelerometry data were included in the current analyses. The 12 excluded participants did not differ significantly from the included participants in terms of age, ethnicity, BMI, fat weight, lean weight, or insulin sensitivity $(P>.40)$.

3.2. Adiponectin, Adiposity, and Insulin Sensitivity. Adiponectin levels (mean $8.7 \pm 3.0 \mu \mathrm{g} / \mathrm{mL}$; range $1.1-14.0 \mu \mathrm{g} / \mathrm{mL}$ ) were normally distributed. Adiponectin did not differ by Tanner stage (Tanner 1, $N=20$, adiponectin $9.4 \pm 2.9 \mu \mathrm{g} / \mathrm{mL}$; Tanner 2, $N=19$, adiponectin $8.0 \pm 3.1 \mu \mathrm{g} / \mathrm{mL} ; P=.16$ ), or differ by ethnicity (Latina, $N=30,8.7 \pm 3.0 \mu \mathrm{g} / \mathrm{mL}$; AfricanAmerican, $N=9,8.8 \pm 3.1 \mu \mathrm{g} / \mathrm{mL} ; P=.89)$.

Table 2 shows correlations between adiponectin and anthropometric measures, clinical measures, and accelerometry data. Adiponectin levels correlated negatively with BMI $(r=-0.41, P=.01)$ and fat weight $(r=-0.43, P<.01)$ and correlated positively with SI $(r=0.52, P<.01)$.

\subsection{Relationship to Physical Activity and Sedentary Behavior.} Table 3 shows the results of linear regression analysis for the outcome adiponectin. After adjusting for age, fat weight, lean weight, and SI, mean counts per minute $(\beta=-0.36$, $P=.02)$, mean minutes spent in DPA $(\beta=-0.40$, $P=.02)$, mean minutes spent in MPA $(\beta=-0.36$, $P=.04)$, or mean minutes spent in MVPA $(\beta=-0.37$, $P=.03)$ were significant predictors of adiponectin levels. The results for VPA $(\beta=-0.28, P=.08)$ trended towards significance. There was no association between mean minutes spent in SB and adiponectin levels in the adjusted model $(\beta=0.23, P=.13)$. Insulin sensitivity was a significant predictor of adiponectin levels in all models $(P<.05)$.

Results were stratified by BMI percentile to explore if the relationship between adiponectin and PA was different in nonoverweight versus overweight children. In participants with BMIs $\geq 85$ th percentile $(N=22)$, after adjusting for age, fat weight, lean weight, and SI, mean minutes spent in DPA ( $\beta=-0.49, P=.04)$ was a significant predictor of adiponectin levels. Mean minutes spent in $\mathrm{SB}(\beta=0.06$, $P=.81)$, MPA $(\beta=-0.36, P=.12)$, VPA $(\beta=0.000$, $P=1.0)$, or MVPA $(\beta=-0.33, P=.15)$ were not significant predictors of adiponectin levels. In participants with BMIs $<$ 85 th percentile $(N=17)$, after adjusting for age, fat weight, lean weight, and SI, mean minutes spent in DPA $(\beta=0.16$, $P=.58), \mathrm{SB}(\beta=0.20, P=.34)$, MPA $(\beta=0.22, P=.48)$, VPA $(\beta=0.19, P=0.55)$, or MVPA $(\beta=0.26, P=0.58)$ were not significant predictors of adiponectin levels. 
TABle 1: Subject characteristics $(N=39)$.

\begin{tabular}{|c|c|}
\hline Variable & \\
\hline Anthropometric/Demographic Measures & \\
\hline Age (years) & $9.2 \pm 0.9$ \\
\hline Ethnicity (\% Latina) & $77 \%$ \\
\hline BMI $\left(\mathrm{kg} / \mathrm{m}^{2}\right)$ & $21.9 \pm 4.9$ \\
\hline BMI percentile (CDC guidelines) & $79.6 \pm 23.8$ \\
\hline BMI percentile $\geq 85 \%$ (overweight/obese; $\%$ meeting criteria) & $56 \%$ \\
\hline Fat weight $(\mathrm{kg})$ & $12.8 \pm 8.6$ \\
\hline Fat weight ( $\%$ of total weight) & $26.4 \pm 10.9$ \\
\hline Lean weight $(\mathrm{kg})$ & $31.0 \pm 7.1$ \\
\hline Clinical Measures & \\
\hline Adiponectin $(\mu \mathrm{g} / \mathrm{mL})$ & $8.7 \pm 3.0$ \\
\hline Fasting insulin $(\mu \mathrm{U} / \mathrm{mL})$ & $15.0 \pm 13.0$ \\
\hline Fasting glucose $(\mathrm{mg} / \mathrm{dL})$ & $92.0 \pm 5.2$ \\
\hline Insulin sensitivity $(\mathrm{SI})\left(\times 10^{-4} \mathrm{~min}^{-1} /(\mu \mathrm{IU} / \mathrm{mL})\right)$ & $3.3 \pm 2.0$ \\
\hline Acute insulin response to glucose $(\mathrm{AIRg})(\mu \mathrm{U} / \mathrm{mL} \times \min )$ & $1196 \pm 882$ \\
\hline Accelerometry & \\
\hline Days of valid wear & $6.6 \pm 2.5$ \\
\hline Mean counts per minute & $444 \pm 156$ \\
\hline Minutes/day spent in: & \\
\hline SB & $417 \pm 72$ \\
\hline MPA (4-7 METs) & $44 \pm 24$ \\
\hline VPA ( $\geq 7$ METs $)$ & $4 \pm 7$ \\
\hline MVPA ( $\geq 4$ METs) & $48 \pm 29$ \\
\hline DPA ( $\geq 3$ METs) & $122 \pm 47$ \\
\hline
\end{tabular}

Values shown are mean \pm standard deviation

BMI: Body mass index, CDC: Centers for Disease Control and Prevention, METs: Metabolic equivalents, PA: Physical activity, SB: Sedentary behavior, MPA: Moderate PA, VPA: Vigorous PA, MVPA: Moderate-to-vigorous PA, and DPA: Daily PA.

TABLE 2: Unadjusted correlations between adiponectin and anthropometric and clinical measures.

\begin{tabular}{lcc}
\hline Variable & $r$ & $P$-value \\
\hline Anthropometric/Demographic Measures & -0.21 \\
Age $($ years $)$ & -0.41 & 0.20 \\
BMI $\left(\mathrm{kg} / \mathrm{m}^{2}\right)$ & -0.43 & $0.01^{*}$ \\
Fat weight $(\mathrm{kg})$ & -0.26 \\
Lean weight $(\mathrm{kg})$ & -0.43 \\
\hline Clinical Measures & -0.34 \\
Fasting insulin $(\mu \mathrm{IU} / \mathrm{mL})(\log )$ & 0.52 \\
Fasting glucose $(\mathrm{mg} / \mathrm{dL})(\log )$ & -0.57 \\
Insulin sensitivity $(\mathrm{SI})\left(\times 10^{-4} \mathrm{~min}^{-1} /(\mu \mathrm{IU} / \mathrm{mL})\right)$ & $<0.01^{*}$ \\
Acute insulin response to glucose $(\mathrm{AIRg})(\mu \mathrm{U} / \mathrm{mL} \times \mathrm{min})(\log )$ & $0.03^{*}$ \\
\hline Accelerometry & $<0.01^{*}$ \\
Mean counts per minute & -0.04 \\
Minutes/day spent in: & & $<0.001^{*}$ \\
SB & 0.09 \\
MPA $(4-7 \mathrm{METs})$ & 0.02 \\
VPA $(\geq 7$ METs $)$ & 0.02 \\
MVPA $(\geq 4 \mathrm{METs})$ & 0.02 \\
DPA $(\geq 3 \mathrm{METs})$ & -0.002 & 0.81 \\
\hline
\end{tabular}

\footnotetext{
${ }^{*} P<.05$.
}

BMI: Body mass index, METs: Metabolic equivalents, PA: Physical activity, SB: Sedentary behavior, MPA: Moderate PA, VPA: Vigorous PA, MVPA: Moderateto-vigorous PA, and DPA: Daily PA. 
TABLE 3: Linear regression for the outcome adiponectin levels controlling for age, fat weight, lean weight, and insulin sensitivity.

\begin{tabular}{|c|c|c|c|c|c|}
\hline \multirow{2}{*}{ PA variable } & \multicolumn{3}{|c|}{ Overall Model } & \multicolumn{2}{|c|}{ Physical Activity } \\
\hline & $R$ & $R^{2}$ & $P$-value & $\beta^{* *}$ & $P$-value \\
\hline Mean counts per minute & 0.66 & 0.44 & $<0.01^{*}$ & -0.36 & $0.02^{*}$ \\
\hline Minutes/day SB & 0.62 & 0.38 & $<0.01^{*}$ & 0.23 & 0.13 \\
\hline Minutes/day MPA (4-7 METs) & 0.65 & 0.42 & $<0.01^{*}$ & -0.36 & $0.04^{*}$ \\
\hline Minutes/day VPA ( $\geq 7$ METs) & 0.63 & 0.40 & $<0.01^{*}$ & -0.28 & 0.08 \\
\hline Minutes/day MVPA ( $\geq 4$ METs) & 0.65 & 0.43 & $<0.01^{*}$ & -0.37 & $0.03^{*}$ \\
\hline Minutes/day DPA ( $\geq 3$ METs) & 0.66 & 0.44 & $<0.01^{*}$ & -0.40 & $0.02^{*}$ \\
\hline
\end{tabular}

${ }^{*} P<.05$.

$* * \beta$ coefficients are standardized,

METs: Metabolic equivalents, PA: Physical activity, SB: Sedentary behavior, MPA: Moderate PA, MVPA: Moderate-to-vigorous PA, and DPA: Daily PA.

\section{Discussion}

Adiponectin levels correlated positively with SI and negatively with measures of adiposity and AIRg in minority female youth. The relationship between adiponectin and SI has been shown previously using results from the oral glucose tolerance test [12] and the homeostasis model assessment of insulin resistance (HOMA-IR) $[9,11,13,14]$. The measure used here, frequently sampled intravenous glucose tolerance test (FSIVGTT), allows for the estimation of SI as well as beta cell functionality with AIRg. Lower acute insulin response and higher insulin sensitivity may be related in part through adiponectin because insulin can suppress adiponectin levels [26] and adiponectin can increase insulin sensitivity [2].

In linear regression models, more time spent in PA was associated with significantly lower adiponectin levels. The inverse association between PA and adiponectin is somewhat surprising, but consistent with previous studies which have shown a decrease in adiponectin with increasing PA [13, 14] or physical fitness [15]. It has been suggested that the expression or secretion of adiponectin may be reduced by PA-induced improvement in insulin action [27] or that adiponectin levels are downregulated when physical activity levels are sufficient to maintain insulin sensitivity [13]. Alternative mechanisms for such a relationship are upregulation of adiponectin receptors or a shift in the amount of adiponectin protein existing as the more biologically active isoform, high-molecular weight (HMW) adiponectin, with increasing PA.

Adiponectin has two receptors: Adiponectin receptor-1 (AdipoR1) and adiponectin receptor-2 (AdipoR2) [28]. Both of these receptors are expressed in human skeletal muscle [29] and may play a role in the insulin-sensitizing effect of adiponectin [29, 30]. Higher levels of PA could lead to an upregulation of the receptors for adiponectin, resulting in a reduced need for high levels of circulating adiponectin. While a single bout of exercise may either increase [30] or decrease [31] AdipoR1 or AdipoR2 expression levels, long-term exercise interventions appear to increase AdipoR1 and AdipoR2 expression levels in skeletal muscle [30, 32]. This could lead to a decrease in adiponectin protein levels, as adiponectin receptor expression and adiponectin protein levels may be inversely related [30]. However, changes in adiponectin receptor expression levels are not always accompanied by changes in circulating levels of adiponectin protein [32]. Also, it is not known if changes in adiponectin receptor expression level reflect membrane receptor availability.

Rather than altering adiponectin receptors, PA could change the amount of adiponectin existing as the more biologically active isoform, high-molecular weight adiponectin. Adiponectin exists as three multimers: high molecular weight (HMW; >220 kilodaltons (kDa)), middle molecular weight (MMW; $120 \mathrm{kDa}$ ), and low molecular weight (LMW; $\sim 70 \mathrm{kDa}$ ) adiponectin [32]. The HMW form of adiponectin may be more biologically active and more tightly correlated with insulin sensitivity than MMW or LMW [32], and adiponectin isoform levels may be more informative than the total amount of adiponectin [33]. Physical activity may change the ratio of adiponectin multimer isoforms (decrease MMW relative to HMW and LMW) [32], thereby increasing the biological activity of adiponectin.

When results were stratified by weight status, the association between adiponectin and PA was only seen in those participants who were overweight (BMI $\geq 85$ th percentile). Previous research has shown that AdipoR1 expression is higher in obese participants compared to lean controls [34], and that AdipoR1 expression increased in the muscle of obese, but not lean, rats after an 8-week moderate exercise program [35]. Although this finding needs to be replicated in larger studies, the inverse relationship between adiponectin and PA may be stronger in overweight/obese children because of adiponectin receptor expression levels.

There was no significant association between SB and adiponectin levels after adjustment for adiposity and SI. It is possible that the mechanisms that regulate the decrease in adiponectin with PA are not downregulated with increasing $\mathrm{SB}$. However, there was a slight trend towards an increase in adiponectin with increasing $\mathrm{SB}$ in the linear regression ( $\beta=0.23, P=0.13$ ), which would support the hypothesis that adiponectin may be increased to compensate for a lack of PA. This is the first study to evaluate the association between adiponectin and SB and this relationship should be evaluated further. 
This study has several strengths, including an objective measure of physical activity, insulin sensitivity determined by FSIVGTT, and participants from an understudied population. Limitations include the small sample size, crosssectional design which does not allow determination of directionality between two variables particularly when they both can alter over time, very low levels of vigorous PA which makes it difficult to examine the relationship between VPA and adiponectin, and no measure of physical fitness which could be a mediator of the relationship between PA and adiponectin. In addition, studies of PA and adiponectin receptors and isoforms were conducted in adults, and it is unknown if the mechanisms outlined here are applicable to children. These results may not generalize to other populations, including male children, and should be verified using a larger sample size and longitudinal design.

Adiponectin is an important protein because of its association with chronic diseases such as cardiovascular disease, cancer, and diabetes. To the best of our knowledge, this is the first study to examine the association between PA and adiponectin levels in minority children and also the first to examine the relationship between adiponectin and SI as measured by FSIVGTT. Although small, this study does cast doubt upon the hypothesis that adiponectin levels increase with increasing levels of PA independent of changes in body fat and proposes potential mechanisms for such a relationship. Future studies may be able to clarify the mechanism through which adiponectin and PA are related and provide insight into the regulation of this important adipose-derived hormone.

\section{Acknowledgments}

This work was supported by the USC Center for Transdisciplinary Research on Energetics and Cancer (NCI, U54 CA 116848) and the Comprehensive NCMHD Research Center of Excellence (P60 MD 002254). There were no conflicts of interest. The authors would like to thank Ana Romero, Adriana Padilla, Flor Del Hoyo, Arianna McClain, Marc Weigensberg, and Selena Nguyen-Rodriguez for their roles in development, data collection, and management of this project. The authors would also like to thank all of their participants and their families for their participation in this study.

\section{References}

[1] Y. Arita, S. Kihara, N. Ouchi et al., "Paradoxical decrease of an adipose-specific protein, adiponectin, in obesity," Biochemical and Biophysical Research Communications, vol. 257, no. 1, pp. 79-83, 1999.

[2] T. Kadowaki, T. Yamauchi, N. Kubota, K. Hara, K. Ueki, and $\mathrm{K}$. Tobe, "Adiponectin and adiponectin receptors in insulin resistance, diabetes, and the metabolic syndrome," Journal of Clinical Investigation, vol. 116, no. 7, pp. 1784-1792, 2006.

[3] K. A. Simpson and M. A. F. Singh, "Effects of exercise on adiponectin: a systematic review," Obesity, vol. 16, no. 2, pp. 241-256, 2008.
[4] C. L. Ogden, M. D. Carroll, and K. M. Flegal, "High body mass index for age among US children and adolescents, 2003-2006," Journal of the American Medical Association, vol. 299, no. 20, pp. 2401-2405, 2008.

[5] A. N. Jeffery, M. J. Murphy, B. S. Metcalf et al., "Adiponectin in childhood," International Journal of Pediatric Obesity, vol. 3 , no. 3, pp. 130-140, 2008.

[6] C. Antoniades, A. S. Antonopoulos, D. Tousoulis, and C. Stefanadis, "Adiponectin: from obesity to cardiovascular disease: etiology and pathophysiology," Obesity Reviews, vol. 10, no. 3, pp. 269-279, 2009.

[7] I. Kelesidis, T. Kelesidis, and C. S. Mantzoros, "Adiponectin and cancer: a systematic review," British Journal of Cancer, vol. 94, no. 9, pp. 1221-1225, 2006.

[8] S. Li, H. J. Shin, E. L. Ding, and R. M. van Dam, "Adiponectin levels and risk of type 2 diabetes: a systematic review and metaanalysis," Journal of the American Medical Association, vol. 302, no. 2, pp. 179-188, 2009.

[9] P. Balagopal, D. George, H. Yarandi, V. Funanage, and E. Bayne, "Reversal of obesity-related hypoadiponectinemia by lifestyle intervention: a controlled, randomized study in obese adolescents," Journal of Clinical Endocrinology \& Metabolism, vol. 90, no. 11, pp. 6192-6197, 2005.

[10] E. S. Kim, J.-A. Im, K. C. Kim et al., "Improved insulin sensitivity and adiponectin level after exercise training in obese Korean youth," Obesity, vol. 15, no. 12, pp. 3023-3030, 2007.

[11] Z. Punthakee, E. E. Delvin, J. O’Loughlin et al., "Adiponectin, adiposity, and insulin resistance in children and adolescents," Journal of Clinical Endocrinology \& Metabolism, vol. 91, no. 6, pp. 2119-2125, 2006.

[12] G. P. Nassis, K. Papantakou, K. Skenderi et al., "Aerobic exercise training improves insulin sensitivity without changes in body weight, body fat, adiponectin, and inflammatory markers in overweight and obese girls," Metabolism, vol. 54, no. 11, pp. 1472-1479, 2005.

[13] B. S. Metcalf, A. N. Jeffery, J. Hosking, L. D. Voss, N. Sattar, and T. J. Wilkin, "Objectively measured physical activity and its association with adiponectin and other novel metabolic markers," Diabetes Care, vol. 32, no. 3, pp. 467-473, 2009.

[14] C. Platat, A. Wagner, T. Klumpp, B. Schweitzer, and C. Simon, "Relationships of physical activity with metabolic syndrome features and low-grade inflammation in adolescents," Diabetologia, vol. 49, no. 9, pp. 2078-2085, 2006.

[15] D. Nemet, P. Wang, T. Funahashi et al., "Adipocytokines, body composition, and fitness in children," Pediatric Research, vol. 53, no. 1, pp. 148-152, 2003.

[16] R. J. Kuczmarski, C. L. Ogden, S. S. Guo et al., “2000 CDC growth charts for the United States: methods and development," Vital and Health Statistics. Series 11, Data from the National Health Survey, no. 246, pp. 1-190, 2002.

[17] D. A. Fields, M. I. Goran, and M. A. McCrory, "Bodycomposition assessment via air-displacement plethysmography in adults and children: a review," American Journal of Clinical Nutrition, vol. 75, no. 3, pp. 453-467, 2002.

[18] P. J. Gately, D. Radley, C. B. Cooke et al., "Comparison of body composition methods in overweight and obese children," Journal of Applied Physiology, vol. 95, no. 5, pp. 2039-2046, 2003.

[19] R. N. Bergman, Y. Z. Ider, C. R. Bowden, and C. Cobelli, "Quantitative estimation of insulin sensitivity," The American Journal of Physiology, vol. 236, no. 6, pp. E667-E677, 1979. 
[20] G. Pacini and R. N. Bergman, "MINMOD: a computer program to calculate insulin sensitivity and pancreatic responsivity from the frequently sampled intravenous glucose tolerance test," Computer Methods and Programs in Biomedicine, vol. 23, no. 2, pp. 113-122, 1986.

[21] S. G. Trost, R. R. Pate, J. F. Sallis et al., "Age and gender differences in objectively measured physical activity in youth," Medicine \& Science in Sports \& Exercise, vol. 34, no. 2, pp. 350355, 2002.

[22] R. R. Pate, J. Stevens, L. S. Webber et al., "Age-related change in physical activity in adolescent girls," Journal of Adolescent Health, vol. 44, no. 3, pp. 275-282, 2009.

[23] C. E. Matthews, K. Y. Chen, P. S. Freedson et al., "Amount of time spent in sedentary behaviors in the United States, 20032004," American Journal of Epidemiology, vol. 167, no. 7, pp. 875-881, 2008.

[24] M. S. Treuth, K. Schmitz, D. J. Catellier et al., "Defining accelerometer thresholds for activity intensities in adolescent girls," Medicine \& Science in Sports \& Exercise, vol. 36, no. 7, pp. 1259-1266, 2004.

[25] T. J. Cole, M. C. Bellizzi, K. M. Flegal, and W. H. Dietz, "Establishing a standard definition for child overweight and obesity worldwide: international survey," British Medical Journal, vol. 320, no. 7244, pp. 1240-1243, 2000.

[26] M. Möhlig, U. Wegewitz, M. Osterhoff et al., "Insulin decreases human adiponectin plasma levels," Hormone and Metabolic Research, vol. 34, no. 11-12, pp. 655-658, 2002.

[27] T. Yatagai, Y. Nishida, S. Nagasaka et al., "Relationship between exercise training-induced increase in insulin sensitivity and adiponectinemia in healthy men," Endocrine Journal, vol. 50, no. 2, pp. 233-238, 2003.

[28] T. Yamauchi, J. Kamon, Y. Ito et al., "Cloning of adiponectin receptors that mediate antidiabetic metabolic effects," Nature, vol. 423 , no. 6941, pp. 762-769, 2003.

[29] A. E. Civitarese, C. P. Jenkinson, D. Richardson et al., "Adiponectin receptors gene expression and insulin sensitivity in non-diabetic Mexican Americans with or without a family history of type 2 diabetes," Diabetologia, vol. 47, no. 5, pp. 816 820, 2004.

[30] M. Blüher, J. W. Bullen Jr., J. H. Lee et al., "Circulating adiponectin and expression of adiponectin receptors in human skeletal muscle: associations with metabolic parameters and insulin resistance and regulation by physical training," Journal of Clinical Endocrinology \& Metabolism, vol. 91, no. 6, pp. 2310-2316, 2006.

[31] C. Punyadeera, A. H. G. Zorenc, R. Koopman et al., "The effects of exercise and adipose tissue lipolysis on plasma adiponectin concentration and adiponectin receptor expression in human skeletal muscle," European Journal of Endocrinology, vol. 152, no. 3, pp. 427-436, 2005.

[32] V. B. O'Leary, A. E. Jorett, C. M. Marchetti et al., "Enhanced adiponectin multimer ratio and skeletal muscle adiponectin receptor expression following exercise training and diet in older insulin-resistant adults," American Journal of Physiology, vol. 293, no. 1, pp. E421-E427, 2007.

[33] T.-S. Tsao, E. Tomas, H. E. Murrey et al., "Role of disulfide bonds in acrp30/adiponectin structure and signaling specificity. Different oligomers activate different signal transduction pathways," Journal of Biological Chemistry, vol. 278, no. 50, pp. 50810-50817, 2003.

[34] M. B. Chen, A. J. McAinch, S. L. Macaulay et al., "Impaired activation of AMP-kinase and fatty acid oxidation by globular adiponectin in cultured human skeletal muscle of obese type 2 diabetics," Journal of Clinical Endocrinology \& Metabolism, vol. 90, no. 6, pp. 3665-3672, 2005.

[35] S.-P. Chang, Y.-H. Chen, W.-C. Chang, I.-M. Liu, and J.T. Cheng, "Increase of adiponectin receptor gene expression by physical exercise in soleus muscle of obese Zucker rats," European Journal of Applied Physiology, vol. 97, no. 2, pp. 189195, 2006. 